\title{
Responses to Overdiagnosis in Thyroid Cancer Screening among Korean Women
}

Sangeun Lee, $\mathrm{BN}^{1}$
Yoon Young Lee, $\mathrm{MPH}^{2}$
Hyo Joong Yoon, $\mathrm{BS}^{2}$
Eunji Choi, $\mathrm{BA}^{1}$
Mina Suh, MD, PhD²
Boyoung Park, MD, PhD²
Jae Kwan Jun, MD, $\mathrm{PhD}^{1,2}$
Yeol Kim, MD, MPH
Kui Son Choi, PhD',2

${ }^{1}$ Graduate School of Cancer Science and Policy, Goyang, ${ }^{2}$ National Cancer Control Institute, National Cancer Center, Goyang, Korea
Correspondence: Kui Son Choi, PhD Graduate School of Cancer Science and Policy, National Cancer Center, 323 Ilsan-ro, Ilsandong-gu, Goyang 10408, Korea Tel: 82-31-920-2912

Fax: 82-31-920-2189

E-mail: kschoi@ncc.re.kr

Received June 18, 2015

Accepted December 11, 2015

Published Online December 28, 2015

\begin{abstract}
Purpose
Communicating the harms and benefits of thyroid screening is necessary to help individuals decide on whether or not to undergo thyroid cancer screening. This study was conducted to assess changes in thyroid cancer screening intention in response to receiving information about overdiagnosis and to determine factors with the greatest influence thereon.
\end{abstract}

\section{Materials and Methods}

Data were acquired from subjects included in the 2013 Korean National Cancer Screening Survey (KNCSS), a nationwide, population-based, cross-sectional survey. Of the 4,100 respondents in the $2013 \mathrm{KNCSS}$, women were randomly subsampled and an additional face-to-face interview was conducted. Finally, a total of 586 female subjects were included in this study. Intention to undergo thyroid cancer screening was assessed before and after receiving information on overdiagnosis.

\section{Results}

Prior awareness of overdiagnosis in thyroid cancer screening was $27.8 \%$. The majority of subjects intended to undergo thyroid cancer screening before and after receiving information on overdiagnosis ( $87 \%$ and $74 \%$, respectively). Only a small number of subjects changed their intention to undergo thyroid cancer screening from positive to negative after receiving information on overdiagnosis. Women of higher education level and Medical Aid Program recipients reported being significantly more likely to change their intention to undergo thyroid cancer screening after receiving information on overdiagnosis, while women with stronger beliefs on the efficacy of cancer screening were less likely to change their intention.

\section{Conclusion}

Women in Korea appeared to be less concerned about overdiagnosis when deciding whether or not to undergo thyroid cancer screening.

Key words

Medical overuse, Thyroid neoplasms,

Early detection of cancer, Intention

\section{Introduction}

The incidence of thyroid cancer in Korea is rapidly increasing. The age-standardized incidence rate of thyroid cancer in 2012 was 62.5 per 100,000 persons ( 23.0 per 100,000 men and 102.4 per 100,000 women), and was the most common cancer (sixth most common cancer in men and the most common cancer in women) among Koreans [1]. The average annual percentage change in thyroid cancer incidence for 1999-2012 was $22.3 \%$ (23.6\% in men and $22.1 \%$ in women), the most rapid among all cancers in Korea [1]. Nevertheless, despite an increase in incidence, mortality rates have remained nearly constant over the past three decades [2]. 
Increases in thyroid cancer incidence have been reported not only in Korea but also in many other parts of the world. The rate of thyroid cancer diagnosis has more than doubled from 4.9 per 100,000 persons in 1975 to 10.6 per 100,000 persons in 2005; meanwhile, thyroid cancer mortality rates in the United States have remained among the most stable [3]. Although the reasons for the rapid rise in thyroid cancer diagnosis have yet to be determined, several groups have argued that the apparent increases in thyroid cancer do not represent a true increase in disease, but rather the results of a large reservoir of undiagnosed thyroid cancers combined with overdiagnosis of small tumors that will never become clinically significant $[4,5]$.

In Korea, the National Cancer Screening Program (NCSP), which was implemented in 1999, provides nationwide screening services free-of-charge or with a small copayment for gastric, liver, colorectal, breast, and cervical cancers. Although thyroid cancer screening is not covered under the NCSP, healthcare providers typically offer ultrasonographic screening for thyroid cancer as an inexpensive add-on at around 30 to 50 USD [4]. Nevertheless, the offering of thyroid cancer screening with ultrasonography as part of routine health checkups is controversial due to the lack of consensus among experts and intrinsic difficulties in accurately estimating rates of overdiagnosis [6]. Generating morbidity that adds to the societal burden of cancer, in terms of medical, psychological, and economic expense, overdiagnosis and overtreatment of inconsequential disease are the most serious harms associated with early detection of cancer through unwarranted screening [3]. An overdiagnosed person is unlikely to benefit from and may be harmed physically and emotionally by diagnostic procedures and treatments.

Despite the harms of overdiagnosis, only a few studies have been conducted to assess the public's understanding of overdiagnosis and how information on overdiagnosis might affect one's decision to undergo screening. For breast cancer, information on screening typically emphasizes the benefits thereof without mentioning overdiagnosis [7-12]. Recent qualitative studies have found that information on overdiagnosis in breast cancer screening can be difficult for screening-eligible women to understand; in addition, it was often found by women to be surprising and counterintuitive $[13,14]$. Nevertheless, once women are informed of the harms, their intentions or actual uptake of breast cancer screening reportedly decrease $[15,16]$. Thus, informing individuals on the harms of cancer screening has become critically important and necessary $[6,7,11,13,14,17,18]$. Indeed, the United Kingdom has since released breast cancer leaflets describing overdiagnosis as "the main risk of screening" [19].

Optimal strategies for communicating information on overdiagnosis in thyroid cancer screening might be more important than breast cancer screening, because it is yet unclear whether increased screening for early detection of thyroid cancer reduces mortality and evidence on the benefits of thyroid cancer screening is limited. Notwithstanding, among women who had previously undergone cancer screening, many had no knowledge regarding overdiagnosis and had little knowledge regarding the harms of overdiagnosis $[6,13]$. Thus, the aim of this study was to assess changes in screening intention after learning about overdiagnosis among women who had prior experiences with cancer screening and to determine factors with the greatest influence thereon. Intention to undergo thyroid cancer screening was assessed before and after subjects had received information.

\section{Materials and Methods}

\section{Study population}

Data were acquired from subjects included in the 2013 Korean National Cancer Screening Survey (KNCSS), an annual cross-sectional survey conducted to examine screening rates among Koreans for five common cancers (gastric, liver, colorectal, breast, and cervix) through nationally representative random sampling. Men ages 40 to 74 years and women ages 30 to 74 years were selected based on Resident of Registration Population data for July 2013 compiled by Statistics Korea, using multistage random sampling according to sex, age, geographic area, and size of the population per area. Interviewers from a professional research agency went door-to-door to recruit residents, and at least three attempts were made to contact individual residents between September 26 and October 18, 2013. The 2013 KNCSS included 4,100 participants (response rate, 69.3\%).

From the 4,100 participants in the 2013 KNCSS, 1,816 women who had previously undergone cancer screening within the past 2 years were selected. An additional faceto-face interview was conducted to examine how women integrated information on overdiagnosis with their existing understanding of thyroid cancer screening and how they might use such information when deciding on screening in the future. Finally, 586 women who completed the KNCSS interview and the additional face-to-face interview were included in this study (32.3\%). Descriptive data for the 586 women included in the study and 1,230 women who were not showed no difference in age, education level, monthly household income, and type of health insurance. The study was approved by the Institutional Review Board of National Cancer Center, Korea, and written informed consent was obtained from all study participants. 


\section{Measures}

The main outcome measure was change in intention to undergo future thyroid screening, which was assessed before and after subjects had received information on overdiagnosis. Before discussing information on overdiagnosis, we briefly described thyroid cancer screening as "Ultrasonographic tests can be used to detect thyroid cancer at early stages in asymptomatic women, the cost of which is around 30 to 50 USD," and assessed the participants' intentions to undergo thyroid cancer screening via a face-to-face interview and in writing in the form of a questionnaire. Thereafter, we gave a brief explanation of overdiagnosis, including a statement on the harms of thyroid cancer screening: "Early stage thyroid cancers detected through screening are usually tiny papillary thyroid tumors, which are very slow-growing, highly unlikely to go on to cause symptoms, much less death. However, thyroid cancer screening could lead to unnecessary thyroidectomy upon diagnosis of thyroid cancer that may not go on to cause symptoms or death in your lifetime." Participants were then asked to respond to this statement to elucidate changes in their intentions to undergo future thyroid cancer screening. To avoid misunderstanding of overdiagnosis among the interviewees, each word in the explanation was chosen carefully by experts in light of previous research. No estimated numbers, such as overdiagnosis rates, were used, since these have not yet been definitively agreed upon. Possible responses included, "I will definitely undergo screening"; "I will probably undergo screening"; "I will probably not undergo screening"; and "I definitely will not undergo screening." The first two answers were considered as positive intentions to undergo screening, while the last two answers were considered as reflecting negative intentions. Changes in intention were coded as a "decrease" or "no decrease."

Characteristics of the study population, including age, household income, insurance coverage, education level, marital status, private cancer insurance, prior awareness of overdiagnosis, thyroid screening history, beliefs on cancer screening efficacy, and family history of cancer (first-degree relatives with any cancer) were also assessed. Insurance coverage was categorized as either National Health Insurance (NHI) or Medical Aid Program (MAP) coverage. Prior awareness of overdiagnosis was measured with the question, "Have you ever heard about the possibility for adverse effects related to overdiagnosis from cancer screening?" and answers of "yes" or "no." Thyroid screening history was restricted to ultrasonography for cancer screening purposes. To measure beliefs on the efficacy of cancer screening, participants were asked to respond to the question, "How effective do you believe cancer screening is in early cancer detection?" on a five-point Likert scale, with one being not effective at all and five being extremely effective. Family history of cancer was limited to that in only blood relatives, including half-siblings.

\section{Statistical analysis}

Univariate and multivariate logistic regression analyses were performed after adjusting for other variables to determine characteristics associated with thyroid cancer screening intention and change therein after receiving information on overdiagnosis. Analyses were performed using STATA ver. 13 (StataCorp. L.P., College Station, TX), and $p<0.05$ was considered statistically significant.

\section{Results}

The baseline characteristics of the study sample $(\mathrm{n}=586)$ are shown in Table 1. The current survey was conducted in women ages 30- to 74-year-old who were invited by the NCSP to undergo cancer screening. Of the 586 respondents, $96 \%$ were NHI beneficiaries, $91 \%$ were married, $86 \%$ were educated to a high-school level or above, and $84 \%$ had private cancer insurance. At baseline, approximately $72 \%$ of subjects had no knowledge regarding overdiagnosis due to cancer screening, and $11 \%$ of subjects had undergone thyroid cancer screening within the past 2 years. Approximately $84 \%$ of subjects thought that cancer screening is effective for early cancer detection, and $27 \%$ had a family history of cancer.

Prior to learning about overdiagnosis, 509 subjects (86.9\%) reported positive intentions (definitely or probably) for undergoing thyroid cancer screening. Simple and multivariate logistic regression analysis was performed to examine predictors of screening intentions before receiving information on overdiagnosis (Table 2). At baseline, subjects with private cancer insurance were more likely to have higher intentions to undergo thyroid cancer screening than those who did not (adjusted odds ratio [aOR], 2.00; 95\% confidence interval [CI], 1.10 to 3.62); none of the other variables were statistically significant.

After receiving information about overdiagnosis, $434 \mathrm{sub}-$ jects $(74.1 \%)$ reported positive intentions (definitely or probably) to undergo thyroid cancer screening (Fig. 1); 428 subjects did not change their positive intentions and six subjects changed their intentions from negative to positive. Among 586 respondents, 505 respondents $(86 \%)$ reported "no decrease" in their intentions: 428 respondents did not change their positive intentions, 71 respondents did not change their negative intentions, and six respondents changed their intentions from negative to positive. Only 
Table 1. Baseline characteristics of the study participants

\begin{tabular}{|c|c|}
\hline Characteristic & No. $(\%)(n=586)$ \\
\hline \multicolumn{2}{|l|}{ Age (yr) } \\
\hline 30-39 & $128(21.8)$ \\
\hline $40-49$ & $152(25.9)$ \\
\hline $50-59$ & $176(30.0)$ \\
\hline $60-74$ & $130(22.2)$ \\
\hline \multicolumn{2}{|l|}{ Education level } \\
\hline Middle school graduate & $85(14.5)$ \\
\hline High school graduate & $347(59.2)$ \\
\hline College and higher & $154(26.3)$ \\
\hline \multicolumn{2}{|l|}{ Monthly household income (US \$) } \\
\hline$\leq 3,499$ & $201(34.3)$ \\
\hline $3,500-4,999$ & $206(35.2)$ \\
\hline$\geq 5,000$ & $179(30.5)$ \\
\hline \multicolumn{2}{|l|}{ Type of health insurance } \\
\hline National Health Insurance & $560(95.6)$ \\
\hline Medical Aid Program & $26(4.4)$ \\
\hline \multicolumn{2}{|l|}{ Private cancer insurance } \\
\hline No & $95(16.2)$ \\
\hline Yes & $491(83.8)$ \\
\hline \multicolumn{2}{|l|}{ Marital status } \\
\hline Single & $51(8.7)$ \\
\hline Married & $535(91.3)$ \\
\hline \multicolumn{2}{|l|}{ Thyroid screening history } \\
\hline $\begin{array}{l}\text { Never screened or not screened } \\
\text { within the past } 2 \text { years }\end{array}$ & $522(89.1)$ \\
\hline Screened within the past 2 years & $64(10.9)$ \\
\hline \multicolumn{2}{|l|}{ Awareness of overdiagnosis } \\
\hline Unaware & $423(72.2)$ \\
\hline Aware & $163(27.8)$ \\
\hline \multicolumn{2}{|l|}{ Family history of cancer } \\
\hline No & $427(72.9)$ \\
\hline Yes & $159(27.1)$ \\
\hline \multicolumn{2}{|l|}{ Belief in cancer screening efficacy } \\
\hline $\begin{array}{l}\text { Totally ineffective/Somewhat } \\
\text { ineffective/Moderate }\end{array}$ & $97(16.5)$ \\
\hline Effective/Very effective & $489(83.5)$ \\
\hline
\end{tabular}

$13.8 \%$ of respondents who showed positive intentions changed their intentions to negative after receiving information about overdiagnosis; this decrease in the percent of women with positive intentions to undergo thyroid cancer screening was statistically significant $(p<0.001)$. Screening intentions and changes in intentions did not differ by age group (Fig. 1).

Finally, logistic regression analysis was performed to determine predictors of decreases in screening intention among the 81 women in the "decrease" group and the 505 women in the "no decrease" group (Table 3). A decrease in intention to undergo screening was more likely in women who graduated high school (aOR, 2.82; 95\% CI, 1.09 to 7.35) and MAP recipients (aOR, 4.29; 95\% CI, 1.58 to 11.63). Women with stronger beliefs on the efficacy of cancer screening were less likely to change their intentions ( $\mathrm{aOR}, 0.37 ; 95 \%$ $\mathrm{CI}, 0.21$ to 0.65 ). Interestingly, prior awareness of overdiagnosis and previous history of thyroid cancer screening was not significantly associated with change in intention.

\section{Discussion}

Among study participants, prior awareness of overdiagnosis in thyroid cancer screening was $27.8 \%$, even though all subjects had undergone at least one cancer screening within the past 2 years. Prior to receiving information on overdiagnosis, $87 \%$ of the subjects intended to undergo thyroid cancer screening, and even after receiving information about overdiagnosis, the majority of women still intended to undergo thyroid cancer screening $(74 \%)$. This finding suggests that women would be willing to undergo screening for thyroid cancer regardless of the risk of overdiagnosis. Only a small number of subjects changed their intentions to undergo thyroid cancer screening from positive to negative after receiving information on overdiagnosis (14\%). Although only $14 \%$ of subjects lowered their intentions to undergo thyroid cancer screening after learning about overdiagnosis, the percentage of women with negative intentions (probably not or definitely not) thereafter was almost double that at baseline $(26 \%$ vs. $13 \%)$. These results suggest that information on overdiagnosis would have an impact on women in the general public, whereas those in the nationwide cancer screening program may have already formed stable beliefs and intentions. This is consistent with previous studies reporting that women who were provided with decision aids were more knowledgeable about screening and demonstrated decreased intentions to undergo screening or were less likely to undergo mammography $[15,16]$.

In the current study, most of the sociodemographic characteristics, including age and income level, were not associated with screening intention, regardless of receiving information on overdiagnosis. However, women who graduated from at least high school were more likely to decrease their intentions to undergo screening. This suggests that the concept of overdiagnosis in itself may have been difficult for the less educated women to understand and that the brief explanation provided to participants in this study may not have been adequate for them to make an informed choice. This result suggests the need for better ways to communicate the importance and risks of overdiagnosis. A recent study 
Table 2. Simple and multiple logistic regression analysis of intentions to undergo thyroid cancer screening prior to receiving information on overdiagnosis

\begin{tabular}{|c|c|c|c|c|}
\hline \multirow{2}{*}{ Variable } & \multicolumn{2}{|c|}{ Thyroid screening intention, No. $(\%)$} & \multirow{2}{*}{$\begin{array}{l}\text { Simple odds } \\
\text { OR }(95 \% \mathrm{CI})\end{array}$} & \multirow{2}{*}{$\begin{array}{l}\text { Multiple odds } \\
\text { aOR }(95 \% \mathrm{CI})\end{array}$} \\
\hline & $\mathrm{Yes}^{\mathrm{a})}(\mathrm{n}=509)$ & $\mathrm{No}^{\mathrm{b})}(\mathrm{n}=77)$ & & \\
\hline \multicolumn{5}{|l|}{ Age (yr) } \\
\hline 30-39 & $107(83.6)$ & $21(16.4)$ & 1 & 1 \\
\hline $40-49$ & $138(90.8)$ & $14(9.2)$ & $1.93(0.94-3.98)$ & $1.88(0.90-3.89)$ \\
\hline $50-59$ & $151(85.8)$ & $25(14.2)$ & $1.19(0.63-2.23)$ & $1.12(0.58-2.15)$ \\
\hline $60-74$ & $113(86.9)$ & $17(13.1)$ & $1.30(0.65-2.61)$ & $1.31(0.57-2.95)$ \\
\hline \multicolumn{5}{|l|}{ Education level } \\
\hline Middle school graduate & $74(87.1)$ & $11(12.9)$ & 1 & 1 \\
\hline High school graduate & $301(86.7)$ & $46(13.3)$ & $0.97(0.48-1.97)$ & $0.90(0.39-2.05)$ \\
\hline College and higher & $134(87.0)$ & $20(13.0)$ & $1.00(0.45-2.19)$ & $0.86(0.33-2.25)$ \\
\hline \multicolumn{5}{|l|}{ Monthly household income (US \$) } \\
\hline$\leq 3,499$ & $174(86.6)$ & $27(13.4)$ & 1 & 1 \\
\hline $3,500-4,999$ & $176(85.4)$ & $30(14.6)$ & $0.91(0.52-1.59)$ & $0.85(0.47-1.56)$ \\
\hline$\geq 5,000$ & $159(88.8)$ & $20(11.2)$ & $1.23(0.67-2.29)$ & $1.14(0.59-2.20)$ \\
\hline \multicolumn{5}{|l|}{ Type of health insurance } \\
\hline National Health Insurance & $487(87.0)$ & $73(13.0)$ & 1 & 1 \\
\hline Medical Aid Program & $22(84.6)$ & $4(15.4)$ & $0.82(0.28-2.46)$ & $0.71(0.22-2.33)$ \\
\hline \multicolumn{5}{|l|}{ Private cancer insurance } \\
\hline No & $76(80.0)$ & $19(20.0)$ & 1 & 1 \\
\hline Yes & $433(88.2)$ & $58(11.8)$ & $1.87(1.05-3.31)$ & $2.00(1.10-3.62)$ \\
\hline \multicolumn{5}{|l|}{ Thyroid screening history within past 2 years } \\
\hline No & $450(86.2)$ & $72(13.8)$ & 1 & 1 \\
\hline Yes & $59(92.2)$ & $5(7.8)$ & $1.89(0.73-4.86)$ & $1.85(0.70-4.87)$ \\
\hline \multicolumn{5}{|l|}{ Awareness of overdiagnosis } \\
\hline Unaware & $361(85.3)$ & $62(14.7)$ & 1 & 1 \\
\hline Aware & $148(90.8)$ & $15(9.2)$ & $1.69(0.93-3.07)$ & $1.80(0.98-3.32)$ \\
\hline \multicolumn{5}{|l|}{ Family history of cancer } \\
\hline No & $366(85.7)$ & $61(14.3)$ & 1 & 1 \\
\hline Yes & $143(89.9)$ & $16(10.1)$ & $1.49(0.83-2.67)$ & $1.49(0.82-2.70)$ \\
\hline \multicolumn{5}{|l|}{ Belief in cancer screening efficacy } \\
\hline Totally ineffective/Somewhat ineffective/Moderate & $86(88.7)$ & $11(11.3)$ & 1 & 1 \\
\hline Effective/Very effective & $423(86.5)$ & $66(13.5)$ & $0.82(0.42-1.62)$ & $0.74(0.37-1.48)$ \\
\hline
\end{tabular}

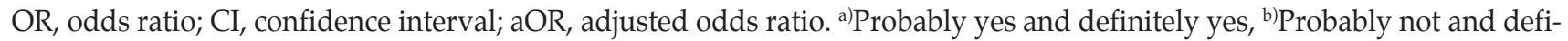
nitely not.

reported that expressing information as a ratio of lives saved to overdiagnosis (1:3) was associated with a greater decrease in intentions to undergo screening than other information formats [6]. In addition, an Australian study also reported that women sometimes confused "overdiagnosis" with "overtreatment," and they were more likely to use "overtreatment" than "overdiagnosis" [13]. In a similar context, one study proposed that some pre-malignant conditions should not be labeled as cancers or neoplasia [17]. Thus, further research is needed to determine methods that would be most helpful to lay individuals in conceptualizing overdiagnosis in Korean women.
In addition, we found that MAP recipients were 4.3 times more likely to lower their intentions to undergo screening after receiving information on overdiagnosis. This might be explained by hesitation to spend money on screening that could ultimately prove unnecessary. In other words, NHI beneficiaries may not have much concern about negative aspects of screening including health care cost due to unnecessary cancer diagnosis and subsequent procedures. This result suggests that an individual's values and priorities may affect their decision, particularly when the benefits of an intervention do not apparently outweigh the harms or when evidence supporting an intervention is not clear with scien- 


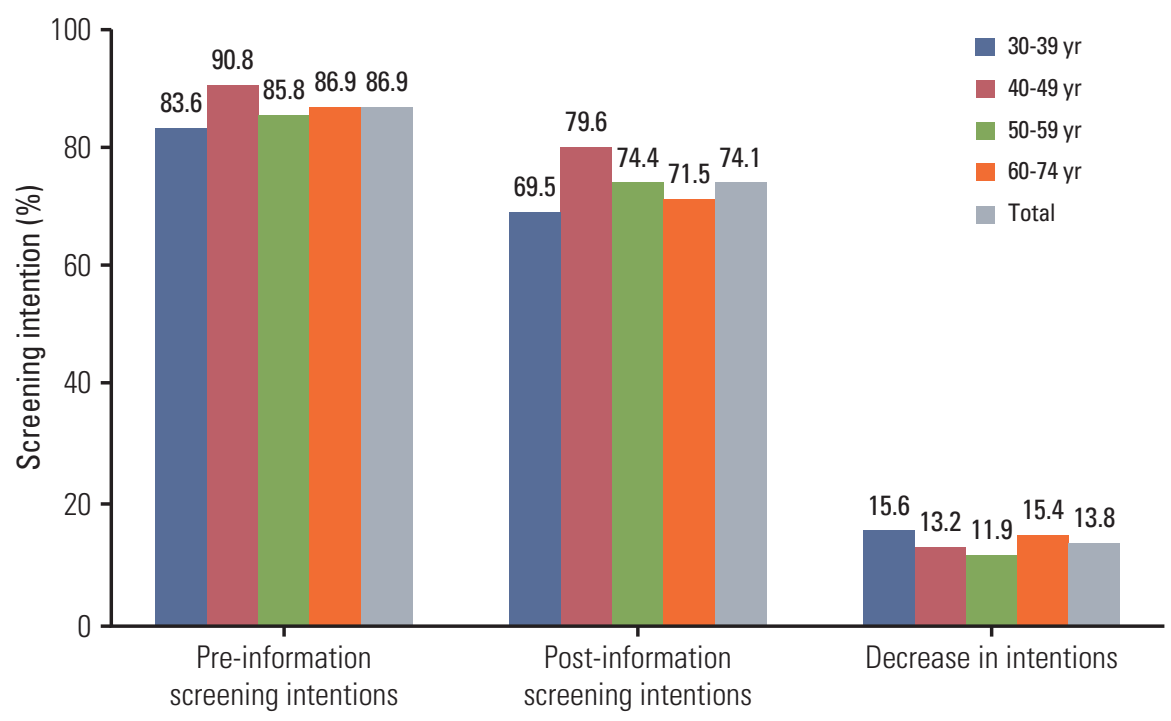

Fig. 1. Positive intention to undergo thyroid cancer screening before and after receiving information on overdiagnosis, stratified by age.

tific certainty [20]. Obviously, accurate information on the benefits and harms of any procedure is important to making an informed decision. However, sometimes, one's beliefs and values regarding their health could be a stronger decisive factor than factual knowledge in informed decision making for cancer screening [21].

In the current study, women who strongly believed that cancer screening is effective in facilitating early detection were less likely to change their intentions to under screening for thyroid cancer. In a recent study on breast cancer screening, women eligible for breast cancer screening via a nationwide screening program reported less of a decrease in their intentions to undergo continued screening after receiving information on overdiagnosis, compared to women not eligible for screening due to younger age [6]. According to another study conducted in Korea, women with strong beliefs on the efficacy of cancer screening underwent thyroid cancer screening 1.55 times more than those who did not [22]. These results were explained by the possibility that eligible women in the screening program might have held strong, stable beliefs on the efficacy of the cancer screening program. Many women seem to believe that screening is extremely important for early detection and treatment of cancer, regardless of its likelihood of becoming malignant. In general, women's attitudes toward cancer screening are shaped through the information presented to them directly by screening service providers, as well as their broader experiences with public health campaigns that promote the benefits of screening without explaining the harms of overdiagnosis.
Thus, one would expect that new information confounding this message (i.e., information about overdiagnosis) would not be immediately accepted and understood, as seen in our study.

There are several limitations to the current study. Subjects were provided only a short amount of time to process the information provided, thus our findings demonstrate only the immediate impact of exposure to information on overdiagnosis [6]. As well, the little amount of information that was written on the questionnaire form and verbally explained by a professional interviewer may not have been adequate to facilitate making an informed choice, and therefore, may have had an impact on our findings. According to previous studies on both written and verbal information, probabilistic information presented as numbers rather than words may provide a more accurate understanding of risk $[18,23]$. In a breast cancer study, numeric information with small numbers appeared to facilitate better understanding [6]. Nevertheless, there is no research on overdiagnosis in thyroid cancer, and no agreement has been reached on estimates of overdiagnosis in thyroid cancer. Thus, we could not provide subjects with probabilistic information presented as numbers, concerning overdiagnosis in thyroid cancer. In addition, we only planned to assess screening intentions rather than actual screening uptake. Future research is needed to assess how information on overdiagnosis works on actual screening uptake. Finally, this study only included women who had previous experiences with cancer screening within the past 2 years, and only $32.3 \%$ of women $(586 / 1,816)$ 
Table 3. Simple and multiple logistic regression analysis of correlates of a decreased intention to undergo thyroid cancer screening (pre-post information)

\begin{tabular}{|c|c|c|c|c|}
\hline \multirow[b]{2}{*}{ Variable } & \multicolumn{2}{|c|}{ Change in intention, No. $(\%)$} & \multirow{2}{*}{$\begin{array}{l}\text { Simple odds } \\
\text { OR }(95 \% \text { CI) }\end{array}$} & \multirow{2}{*}{$\begin{array}{r}\text { Multiple odds } \\
\text { aOR }(95 \% \mathrm{CI})\end{array}$} \\
\hline & $\begin{array}{c}\left.\text { No decrease }^{a}\right) \\
(n=505)\end{array}$ & $\begin{array}{c}\text { Decrease }^{b)} \\
(n=81)\end{array}$ & & \\
\hline \multicolumn{5}{|l|}{ Age (yr) } \\
\hline $30-39$ & $108(84.4)$ & $20(15.6)$ & 1 & 1 \\
\hline $40-49$ & $132(86.8)$ & $20(13.2)$ & $0.82(0.42-1.60)$ & $0.86(0.43-1.72)$ \\
\hline $50-59$ & $155(88.1)$ & $21(11.9)$ & $0.73(0.38-1.42)$ & $0.69(0.35-1.38)$ \\
\hline 60-74 & $110(84.6)$ & $20(15.4)$ & $0.98(0.50-1.93)$ & $1.50(0.68-3.34)$ \\
\hline \multicolumn{5}{|l|}{ Education level } \\
\hline Middle school graduate & $78(91.8)$ & $7(8.2)$ & 1 & 1 \\
\hline High school graduate & $292(84.1)$ & $55(15.9)$ & $2.10(0.92-4.79)$ & $2.82(1.09-7.35)$ \\
\hline College and higher & $135(87.7)$ & $19(12.3)$ & $1.57(0.63-3.90)$ & $2.01(0.67-6.01)$ \\
\hline \multicolumn{5}{|l|}{ Monthly household income (US \$) } \\
\hline$\leq 3,499$ & $173(86.1)$ & $28(13.9)$ & 1 & 1 \\
\hline $3,500-4,999$ & $180(87.4)$ & $26(12.6)$ & $0.89(0.50-1.58)$ & $1.20(0.63-2.30)$ \\
\hline$\geq 5,000$ & $152(84.9)$ & $27(15.1)$ & $1.10(0.62-1.94)$ & $1.47(0.77-2.83)$ \\
\hline \multicolumn{5}{|l|}{ Type of health insurance } \\
\hline National Health Insurance & $488(87.1)$ & $72(12.9)$ & 1 & 1 \\
\hline Medical Aid Program & $17(65.4)$ & $9(34.6)$ & $3.59(1.54-8.35)$ & $4.29(1.58-11.63)$ \\
\hline \multicolumn{5}{|l|}{ Private cancer insurance } \\
\hline No & $86(90.5)$ & $9(9.5)$ & 1 & 1 \\
\hline Yes & $419(85.3)$ & $72(14.7)$ & $1.64(0.79-3.41)$ & $1.50(0.70-3.19)$ \\
\hline \multicolumn{5}{|l|}{ Thyroid screening history within the past 2 years } \\
\hline No & $451(86.4)$ & $71(13.6)$ & 1 & 1 \\
\hline Yes & $54(84.4)$ & $10(15.6)$ & $1.18(0.57-2.42)$ & $1.15(0.54-2.47)$ \\
\hline \multicolumn{5}{|l|}{ Awareness of overdiagnosis } \\
\hline Unaware & $365(86.3)$ & $58(13.7)$ & 1 & 1 \\
\hline Aware & $140(85.9)$ & $23(14.1)$ & $1.03(0.61-1.74)$ & $1.14(0.66-1.97)$ \\
\hline \multicolumn{5}{|l|}{ Family history of cancer } \\
\hline No & $360(84.3)$ & $67(15.7)$ & 1 & 1 \\
\hline Yes & $145(91.2)$ & $14(8.8)$ & $0.52(0.28-0.95)$ & $0.55(0.30-1.03)$ \\
\hline \multicolumn{5}{|l|}{ Belief in cancer screening efficacy } \\
\hline Totally ineffective/Somewhat ineffective/Moderate & $72(74.2)$ & $25(25.8)$ & 1 & 1 \\
\hline Effective/Very effective & $433(88.6)$ & $56(11.4)$ & $0.37(0.22-0.63)$ & $0.37(0.21-0.65)$ \\
\hline
\end{tabular}

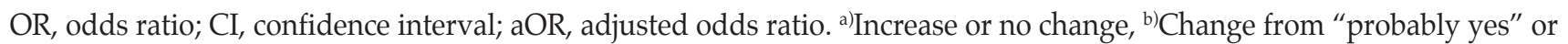
"definitely yes" to "probably not" or "definitely not."

who completed the KNCSS interview and the additional face-to-face interview were included in this study. Although there were no significant differences in sociodemographic characteristics between women included in the study and those who were not, there remains the possibility of selection bias. That is, women with lower intention to undergo thyroid cancer screening might have been less likely to participate in this study. Thus, future studies are needed to investigate thyroid cancer screening intention and awareness of overdiagnosis within the entire general population.

\section{Conclusion}

Early detection of cancer is proven to save lives; however, in some instances it can be harmful, such as those related to overdiagnosis. While women in Korea appeared to be less concerned about overdiagnosis, information on overdiagnosis in thyroid cancer screening in Korea should be provided to individuals so that they can make an informed choice. Herein, the briefest of information resulted in small reductions in intentions to undergo screening for thyroid cancer. 
Nevertheless, providing written information alone may not be helpful to individuals in reaching a full understanding of the harms or benefits of thyroid cancer screening and thereby counteract the Korean public's largely positive attitudes on cancer screening in general. Further research on the relationships between sociodemographic factors and attitudes on cancer screening may be helpful in development of materials for educating the general public on the harms and benefits of thyroid cancer screening.

\section{Conflicts of Interest}

Conflict of interest relevant to this article was not reported.

\section{Acknowledgments}

This study was supported by a Grant-in-Aid for Cancer Research and Control from the National Cancer Center, Korea (Grant number: 1310231).

\section{References}

1. Jung KW, Won YJ, Kong HJ, Oh CM, Cho H, Lee DH, et al. Cancer statistics in Korea: incidence, mortality, survival, and prevalence in 2012. Cancer Res Treat. 2015;47:127-41.

2. Cho BY, Choi HS, Park YJ, Lim JA, Ahn HY, Lee EK, et al. Changes in the clinicopathological characteristics and outcomes of thyroid cancer in Korea over the past four decades. Thyroid. 2013;23:797-804.

3. Welch HG, Black WC. Overdiagnosis in cancer. J Natl Cancer Inst. 2010;102:605-13.

4. Ahn HS, Kim HJ, Welch HG. Korea's thyroid-cancer "epidemic": screening and overdiagnosis. N Engl J Med. 2014;371: 1765-7.

5. Lee JH, Shin SW. Overdiagnosis and screening for thyroid cancer in Korea. Lancet. 2014;384:1848.

6. Waller J, Whitaker KL, Winstanley K, Power E, Wardle J. A survey study of women's responses to information about overdiagnosis in breast cancer screening in Britain. Br J Cancer. 2014;111:1831-5.

7. Gummersbach E, Piccoliori G, Zerbe CO, Altiner A, Othman $\mathrm{C}$, Rose $\mathrm{C}$, et al. Are women getting relevant information about mammography screening for an informed consent: a critical appraisal of information brochures used for screening invitation in Germany, Italy, Spain and France. Eur J Public Health. 2010;20:409-14.

8. Saalasti-Koskinen U, Makela M, Saarenmaa I, Autti-Ramo I. Personal invitations for population-based breast cancer screening. Acad Radiol. 2009;16:546-50.

9. Zapka JG, Geller BM, Bulliard JL, Fracheboud J, Sancho-Garnier $\mathrm{H}$, Ballard-Barbash R. Print information to inform decisions about mammography screening participation in 16 countries with population-based programs. Patient Educ Couns. 2006;63:126-37.

10. Bekker HL. Decision aids and uptake of screening. BMJ. 2010;341:c5407.

11. Duffy SW, Tabar L, Chen TH, Yen AM, Dean PB, Smith RA. What information should be given to women invited for mammographic screening for breast cancer? Womens Health (Lond
Engl). 2006;2:829-33.

12. Jorgensen KJ, Gotzsche PC. Content of invitations for publicly funded screening mammography. BMJ. 2006;332:538-41.

13. Hersch J, Jansen J, Barratt A, Irwig L, Houssami N, Howard $\mathrm{K}$, et al. Women's views on overdiagnosis in breast cancer screening: a qualitative study. BMJ. 2013;346:f158.

14. Waller J, Douglas E, Whitaker KL, Wardle J. Women's responses to information about overdiagnosis in the UK breast cancer screening programme: a qualitative study. BMJ Open. 2013;3:e002703.

15. Mathieu E, Barratt AL, McGeechan K, Davey HM, Howard K, Houssami N. Helping women make choices about mammography screening: an online randomized trial of a decision aid for 40-year-old women. Patient Educ Couns. 2010;81:63-72.

16. Schonberg MA, Hamel MB, Davis RB, Griggs MC, Wee CC, Fagerlin A, et al. Development and evaluation of a decision aid on mammography screening for women 75 years and older. JAMA Intern Med. 2014;174:417-24.

17. Esserman LJ, Thompson IM Jr, Reid B. Overdiagnosis and overtreatment in cancer: an opportunity for improvement. JAMA. 2013;310:797-8.

18. Trevena LJ, Zikmund-Fisher BJ, Edwards A, Gaissmaier W, Galesic M, Han PK, et al. Presenting quantitative information about decision outcomes: a risk communication primer for patient decision aid developers. BMC Med Inform Decis Mak. 2013;13 Suppl 2:S7.

19. Hersch J, Barratt A, Jansen J, Houssami N, Irwig L, Jacklyn G, et al. The effect of information about overdetection of breast cancer on women's decision-making about mammography screening: study protocol for a randomised controlled trial. BMJ Open. 2014;4:e004990.

20. Rimer BK, Briss PA, Zeller PK, Chan EC, Woolf SH. Informed decision making: what is its role in cancer screening? Cancer. 2004;101(5 Suppl):1214-28.

21. Reyna VF, Nelson WL, Han PK, Pignone MP. Decision making and cancer. Am Psychol. 2015;70:105-18.

22. Han MA, Choi KS, Lee HY, Kim Y, Jun JK, Park EC. Current 
status of thyroid cancer screening in Korea: results from a nationwide interview survey. Asian Pac J Cancer Prev. 2011; $12: 1657-63$
23. Trevena LJ, Davey HM, Barratt A, Butow P, Caldwell P. A systematic review on communicating with patients about evidence. J Eval Clin Pract. 2006;12:13-23. 